\title{
Labyrinthe
}

4 | 1999

Numéro 4

\section{Il parle le français comme un cheval allemand...}

Traduit du danois par Priscille Ducet

\section{Ludvig Holberg}

\section{CpenEdition}

Journals

Édition électronique

URL : http://journals.openedition.org/labyrinthe/295

DOI : 10.4000/labyrinthe.295

ISSN : 1950-6031

Éditeur

Hermann

\section{Édition imprimée}

Date de publication : 1 octobre 1999

Pagination : 103-116

\section{Référence électronique}

Ludvig Holberg, « II parle le français comme un cheval allemand... », Labyrinthe [En ligne], 4 | 1999, mis en ligne le 23 août 2005, consulté le 19 avril 2019. URL : http://journals.openedition.org/labyrinthe/295 ; DOI : 10.4000/labyrinthe.295

Ce document a été généré automatiquement le 19 avril 2019

Propriété intellectuelle 


\section{Il parle le français comme un cheval allemand...}

Traduit du danois par Priscille Ducet

Ludvig Holberg

\section{NOTE DE L'ÉDITEUR}

Priscille DuCET est née à Djibouti le 10 mars 1976. Elle est titulaire d'une maitrise de littérature scandinave, préparée conjointement à l'université Paris IV Sorbonne et l'université de Copenhague ; elle travaille actuellement sur le monde souterrain dans la littérature française au XVIII ${ }^{e}$ siècle.

$1 \mathrm{Si}$, aujourd'hui, on dit bien d'une personne s'exprimant fort mal dans une langue étrangère qu'elle " parle comme une vache espagnole ", au XVIII siècle, on disait d'elle qu'elle parle " comme un cheval allemand ». De qui tient-on ce renseignement ? De Ludvig Holberg (1684-1754), le père de la littérature danoise, qui rapporte dans ses Levnedsbreve (lettres autobiographiques) cette anecdote dont il fut lui-même l'objet lorsqu'il se rendit pour la première fois à Paris en 1713. Son français allait néanmoins s'améliorer, puisqu'à son second séjour à Paris en 1725-26, Holberg fut de ceux qui fréquentèrent les cafés littéraires, dont le Café des beaux Esprits, au même titre que JeanBaptiste Rousseau, Houdar de La Motte et surtout Fontenelle, dont il devint l'ami.

Holberg doit tout à la littérature française ; il le dit lui-même dans ses Levnedsbreve. Ses comédies sont en effet bien inspirées du théâtre de Molière : le Fondeur d'étain politicien, la première de ses comédies, est un Bourgeois gentilhomme à la danoise ; l'idée de sa parodie de l'Énéide en vers, Peder Paars, lui a été soufflée par le Lutrin de Boileau ; quant à ses Épitres et Pensées morales, elles portent en elles l'esprit de Montaigne. La France, en revanche, ne le reconnaît plus, et ce en dépit de l'étendue des talents de l'homme, qui écrivit des comédies aussi bien que des traités de droit, d'histoire et de géographie, et une utopie. L'utopie en question a pour titre Le Voyage souterrain de Niels Klim (Niels Klims 
underjordiske Rejse, 1741); Holberg la rédigea en latin afin d'assurer sa diffusion en Europe. $C^{\prime}$ 'est la seule utopie scandinave, et l'une des très rares à illustrer le genre dit du « Voyage souterrain ». Son succès, dès sa parution, fut immédiat ; le roman fut très vite traduit dans toutes les langues européennes - en français par Éléazar de Mauvillon en 1743. Le voyage souterrain de Niels Klim vit néanmoins sa renommée décliner peu à peu, à tel point qu'aujourdhui, aucune traduction moderne du roman n'est disponible. La présente traduction est ainsi inédite, d'une part parce qu'effectuée dans une langue volontairement modernisée, d'autre part dans la mesure où la langue de base est non pas le latin, mais le danois. Il fallut en effet attendre 1789 pour qu'une version danoise du roman de Holberg paraisse ; son maître d'œuvre est Jens Baggesen (1764-1826), également connu dans l'histoire de la littérature danoise pour avoir été l'introducteur de Rousseau auprès de ses compatriotes par le biais de son ouvrage Le Labyrinthe, somme de réflexions directement inspirées des Confessions.

3 Le travail de Baggesen s'apparente néanmoins plus à une adaptation du texte latin qu'à une traduction fidèle, puisque le texte se montre à plusieurs reprises légèrement différent de l'original - un fait qui laisse les savants holbergiens et baggesiens aujourd'hui encore perplexes. C'est pourtant bien cette version danoise que les lycéens et étudiants danois étudient aujourd'hui, lorsque leurs professeurs ne se contentent pas de leur montrer la série télévisée sur les aventures de Niels Klim - une série réalisée avec la participation de la reine Margrete II de Danemark en personne.

Lorsque nous avons appris que certains mettaient en doute l'authenticité de cette histoire, et que l'éditeur du Voyage souterrain avait de ce fait entrepris ici une " mauvaise publication ", nous avons jugé sage de devancer les fausses accusations en dotant les nouvelles publications de témoignages de personnes au-dessus de tout soupçon, et dont la déposition préserve donc l'éditeur des critiques. Nous savons que plusieurs de ces témoins ont été des contemporains de notre héros, les autres ayant pour leur part vécu dans des temps proches; et tout le monde s'entend pour reconnaître les célèbres honnêteté et vertu qui les distinguent et que personne ne leur conteste, pas même l'individu le plus naï. Grâce au témoignage que ces excellentes personnes ont signé de leur propre main, nous faisons taire tous les mauvais juges et les langues fourchues, et les forçons à reconnaître leur erreur et à revenir sur leurs accusations inconsidérées. L'attestation que mon frère et moi avons reçue est conçue comme suit :

Suite à la demande des honnêtes jeunes gens que sont Peder et Andreas Klim, nous certifions avoir trouvé parmi les œuvres posthumes du célèbre Niels Klim un manuscrit intitulé Voyage souterrain ; au même Voyage étaient joints une grammaire souterraine et un dictionnaire en plusieurs langues, en l'occurrence danois et qvamitique. Nous avons comparé ce vieux manuscrit avec la version latine du célèbre Abelin, (que chacun a aujourd'hui en sa possession) et nous affirmons que la traduction ne s'écarte en aucune manière de l'original. Pour l'attester une fois pour toutes, nous apposons ici nos sceaux.

De sa propre main : Adrian Petersen

De même : Jens Thorlaksen

De même : Svend Klak

De même : Jokum Brander

De même : ( Pour lui et son frère ) Jens Grad

De même : Hieronymus Gils, Écossais.

5 Nous espérons qu'un témoignage irréprochable comme celui-ci suffira à lever tous les doutes ; néanmoins, s'il fallait que certains s'acharnent au point de maintenir leurs 
critiques malgré les preuves, nous nous efforcerons de combattre leur mauvaise foi avec d'autres armes. Tout le monde sait que dans cette vallée de Norvège appelée Finmark, vivent des gens qui, grâce à la magie de la Nature (une science à laquelle les savants des autres nations se sont bien peu intéressés), sont capables de réveiller puis d'apaiser une tempête, de se changer en loup, de parler bon nombre de langues dont certaines sont parfaitement étrangères à notre monde, et de se rendre du pôle Sud au pôle Nord en moins d'une heure. Un Finlandais, du nom de Peyvis, s'est récemment rendu à Bergen à la demande du Bourgmestre, et y a montré tant de preuves étranges de sa science et de son art, que l'assemblée tout entière le déclara docteur accompli et émérite ; et comme dans les mêmes temps paraissait une critique acerbe du Voyage souterrain qui qualifiait celuici « d'histoire à dormir debout », le même Peyvis reçut l'ordre de rassembler tout son art pour tenter un voyage sous la terre, car il s'agissait dès lors de laver l'honneur des Klim. Il jura d'obéir à l'ordre, et se répandit alors en paroles sur ses capacités comme suit :

Que veux-tu? Regarde autour de toi ! Ce que la Terre compte de plus grand et de plus fort Depuis l'hémisphère sud jusqu'à l'hémisphère nord Obéit à ma loi. Je suis le gardien des astres et des planètes ; D'un seul geste, j'efface le pont de l'arc-en-ciel ; Le tonnerre ne gronde que si l'envie m'en prend Mer du Nord comme ruisseau, leurs vagues naissent de mes mots J'ai assujetti le vent et la tempête D'un seul mot, je peux changer le roc en miel,

L'Hekkenfeld ${ }^{2}$ en glacier et l'iceberg en volcan.

Que veux-tu ? Regarde autour de toi !

Que n'a-t-on dans la mer, que n'est-il sur la terre,

Du pôle sud au pôle nord, dans le feu et les airs,

Qui n'existe pour moi?

L'assemblée frissonna à l'écoute de ces fantastiques promesses. Mais le Finlandais ne perdit pas plus de temps pour le voyage : il se déshabilla et - ô vision étrange! - se métamorphosa soudain en aigle ; il pris alors son envol et disparut dans les airs. Un vendredi matin, de bonne heure - peu avant le lever du soleil - notre docteur métamorphosable réapparut enfin, après un mois entier d'absence. On le retrouva dans la chaire du bourgmestre de la ville, fourbu comme un cheval ayant battu les campagnes : il était à bout de forces, et la sueur ruisselait sur son front. On le laissa se remettre, et lorsqu'il eut repris son souffle et bu un peu d'eau-de-vie pour reprendre des forces, il commença à décrire son voyage avec soin, et rapporta par bribes ce qu'il avait vécu au cours de sa chevauchée des airs puis des terres souterraines. Il raconta qu'après bien des combats qui avaient cessé depuis, le parti des Klim avait finalement pris le dessus, et la régence était revenue au fils de notre Niels. Celui-ci avait alors longtemps gouverné sous la tutelle de sa mère, mais il régnait à présent en tous lieux dans le monde souterrain, âgé, et encensé pour ses nombreux hauts faits. Tout ce que cet homme averti raconta fut aussitôt mis par écrit mot pour mot. Cela devait devenir un témoignage des plus précieux pour les annales que les Savants avaient promis d'éditer, et qui devaient porter le nom de "Suite à l'histoire de la $V^{e}$ monarchie ». Outre ces annales, une grammaire du qvamitique devait également être mise au point, laquelle ne paraissait alors guère utile même si elle pouvait s'avérer déterminante pour les générations à venir; en effet, notre patrie (sauf modestie de notre part) est une affaire en or pour tous les investisseurs, pourvu qu'ils acceptent de se mettre en quatre pour établir un commerce avec les Qvamites, et de travailler nuit et jour afin de mettre au point des machines capables de descendre sous terre en toute sécurité, et sans l'exercice de la magie. 
7 À votre tour maintenant d'avoir honte, mortels incrédules, et tâchez d'agir dorénavant avec plus de précautions! Ayez honte pour vos langues trop fourchues, et demandez pardon pour vos accusations inconsidérées!

Et ayez honte enfin pour vos critiques impitoyables, et sachez bien que si le monde savant devait ne pas apprécier vos jugements parce qu'ils sont effrontés et irrationnels, alors tuez-les tous donc dans l'œuf, ou, si vous préférez, tuez-vous vous-mêmes.

En l'an mille six cent soixante-quatre, lorsque j'eus passé mes examens à l'université de Copenhague et obtenu la mention laudabilis en théologie comme en philosophie, je fis mes préparatifs pour retourner dans mon pays natal, et j'embarquai à destination de Bergen, en Norvège. J'étais pourvu de certificats excellents, mais dépourvu d'argent, un sort que je partageais avec les autres étudiants norvégiens qui, en général, reviennent la bourse plate du Temple des Muses. Le vent nous était favorable, et après six jours d'une paisible navigation, nous arrivâmes à Bergen, ma ville natale.

Plus instruit, mais pas plus riche, je vécus ensuite quelque temps des subventions de mes parents et de mes proches. Même si je vivais ainsi un peu comme un mendiant, je refusai de passer mon temps à ne rien faire ; et pour approfondir les connaissances en sciences physiques que je m'étais déjà appliqué à acquérir, je décidai d'examiner de plus près le caractère physique du pays et du relief, et je commençai dès lors à errer aux quatre coins de la province. Il n'y avait pas de rocher si escarpé que je ne tente de l'escalader, ni de crevasse assez profonde et sinistre pour me dissuader de l'explorer, dans l'espoir d'y trouver de quoi nourrir l'attention et l'investigation d'un érudit en sciences de la Nature.

Il est certain que dans ma patrie, il existe des choses dont nous-mêmes n'avons personnellement jamais entendu parler, mais qui investiraient le monde savant par des dissertations interminables et nourriraient l'insatiable soif de lecture des médecins, si elles étaient découvertes en France, en Italie, en Allemagne ou dans tout autre lopin de terre propice aux miracles et sachant en faire cas.

Parmi les faits qui me parurent les plus étranges, il y avait une crevasse au sommet d'une montagne que les habitants appelaient « la girouette ». De cette crevasse émane de temps en temps une sorte de vent doux et pas du tout désagréable, comme si le gouffre, en aspirant l'air puis en le réexpulsant, soupirait profondément. Ce soupir a d'ailleurs donné l'occasion aux savants de Bergen notamment le célèbre Abelin et le particulièrement consciencieux Maître Edvard, doyen de l'école latine, d'encourager leurs compatriotes à examiner l'originalité de ce gouffre de plus près, eux-mêmes étant trop vieux et trop faibles pour le faire. Il leur semblait d'autant plus intéressant pour un philosophe méditatif qu'à certains moments, l'air expulsé était réaspiré avec une réelle violence, comme un homme qui ronfle.

Aussi bien motivé par les encouragements de ces grands hommes que par mes propres inclinations naturelles, je méditai une descente dans cette crevasse, et je fis part de mes intentions à quelques bons amis, qui n'en pensèrent rien, sinon qu'il fallait être à la fois fou et désespéré pour tenter une chose pareille. Mais leur réprobation resta parfaitement vaine, et au lieu de décourager cette nouvelle vocation, elle l'encouragea encore davantage. Malgré les risques, j'étais naturellement attiré par les sciences de la Nature, et la situation calamiteuse qui était alors la mienne ne faisait rien pour m'en détourner. Comme j'étais désormais totalement démuni, je supportais de moins en moins de vivre grâce à la miséricorde des autres; et comme le spectre de la pauvreté semblait flotter durablement au-dessus de ma tête, je voyais closes devant moi toutes les portes menant à 
la gloire et à l'ascension dans ma patrie, à moins que je ne me les ouvre en accomplissant quelque chose de sensationnel.

Au terme de ces considérations, j'étais fermement décidé ; et je fis mes préparatifs pour l'expédition; et c'est par un beau temps clair que je partis un jeudi matin, au lever du soleil, dans l'intention d'avoir mené à bien ma tâche avant la nuit. Si j'avais su alors que, tel un nouveau Phaëton, j'allais être

Saisi par la tourmente

D'une tornade sifflante

puis précipité sur une autre planète, et ne devais pas revoir ma patrie et mes amis avant dix années mouvementées!

Cette expédition eut lieu en mille six cent soixante-cinq, à l'époque où Hans Munthe et Lars Sørensen étaient bourgmestres, et Christen Bertelsen et Lars Sand conseillers à Bergen. Je fus accompagné par quatre journaliers portant une corde et un harpon pour mon exploration. Nous nous rendîmes à Sandvig, d'où l'ascension de la montagne est la plus facile, et nous grimpâmes jusqu'au sommet. Nous nous assîmes alors non loin du gouffre fatal pour souffler et prendre le déjeuner qui s'imposait. C'est à ce moment-là seulement que mon cœur commença à tambouriner dans ma poitrine, comme s'il pressentait la catastrophe à venir. Je me tournai vers mes compagnons et demandai : « Lequel d'entre vous veut briser la glace ?" Mais personne ne répondit. La moitié de courage qui s'était tari en moi se raviva alors d'un seul coup ; je les laissai nouer la corde autour de ma taille, je m'assis au bord du gouffre et je recommandai mon âme à Dieu. Alors ils me descendirent dans le précipice, non sans avoir préalablement reçu mes instructions, qui étaient de me faire descendre jusqu'à ce que je crie, auquel signe ils devaient cesser, et si je criais de façon prolongée, de me remonter à toute vitesse. Je pris pour ma part le harpon dans ma main droite pour repousser les membranes que je pouvais rencontrer au cours de ma descente ; et quand je me fus immobilisé au centre du gouffre, je les laissai me descendre. Mais à peine avais-je parcouru dix à douze pieds que la corde cassa. Je poussai alors un cri perçant, tandis que mes quatre compagnons hurlaient en chœur, mais je me mis à dégringoler si vite dans l'abîme que leurs voix ne me parvinrent rapidement plus, et, tel un nouveau Pluto,

Je chutai depuis un entrebâillement de terre

Dans le ventre du gouffre, dont je fendais les airs.

sauf qu'en guise de sceptre, je n'avais qu'un harpon.

18 Je sondais la profonde obscurité d'une nuit interminable depuis environ un quart d'heure - autant que je pouvais en juger dans la situation confuse qui était alors la mienne lorsque je remarquai enfin une lueur, comme un crépuscule, qui fit bientôt place à un ciel clair et incandescent. Je crus d'abord naïvement que j'avais été repoussé vers le haut sous le coup d'un reflux de l'air souterrain ou de quelque autre vent contraire, et que le gouffre m'avait donc réexpiré sur terre. Mais puisque le soleil comme les étoiles que je voyais étaient bien plus petits que ceux que nous connaissons sur terre, je dus me rendre à l'évidence. Cette nouvelle structure céleste était donc soit une création du trouble qui régnait alors dans mon pauvre cerveau, soit j'étais mort et on me dirigeait vers de bienheureuses habitations. Je réalisai toutefois immédiatement combien cette dernière hypothèse était dérisoire lorsque je me vis moi-même, armé d'un harpon et traînant la longue corde derrière moi ; en effet, je me doutais bien qu'une corde et un harpon ne sont nullement nécessaires pour aller au Paradis, et qu'en aucun cas les citoyens célestes ne 
sauraient ressembler à de tels ornements - à moins que mon destin ne fut d'assaillir les cieux pour en chasser les Dieux, comme le firent les Titans. Après avoir gravement médité les faits, je finis par conclure que j'avais échoué sous un ciel souterrain, et que l'hypothèse de ceux qui prétendent que la terre est creuse et qu'elle cache dans son centre un monde plus petit avec un soleil, des planètes et des étoiles plus petits dans des proportions égales, était fondée. L'issue devait montrer que je devinais juste.

La vitesse avec laquelle je chutais était restée constante assez longtemps, jusqu'à ce qu'enfin, je la sente décroître, à mesure que je m'approchais de la planète ou du globe céleste placé en premier sur ma trajectoire de chute. Celui-ci s'agrandit peu à peu, en sorte que je pus bientôt distinguer des lacs, des montagnes et des vallées à travers l'épaisse atmosphère qui l'entourait.

Mon corps était porté par des ailes légères ; J'étais comme l'oiseau qui, entre ciel et terre, S'élève bien au delà des rochers poissonneux, Des vagues turbulentes, et des pics montagneux

Je me rendis bientôt compte que je n'étais pas le seul à planer ainsi dans l'air céleste, mais que la ligne verticale dans laquelle je m'étais enroulé en tombant avait formé un cercle. Je ne nie pas qu'à cette découverte, mes cheveux se dressèrent d'effroi sur ma tête, dans l'effrayante perspective d'être devenu un satellite et d'avoir donc à tourner en orbite jusqu'à la nuit des temps. Toutefois, lorsque je considérai que mon honneur n'était pas pour autant atteint, et que le statut de globe céleste ou de satellite pouvait au moins m'élever au grade de Studiosus philosophiae, je sentis mon courage renaître ; j'étais comme dopé par l'air souterrain et je n'avais ni faim, ni soif. Je me rappelai toutefois que j'avais un morceau de pain dans ma poche ; je le sortis pour voir si, dans mon état d'alors, je pouvais y prendre goût, mais à la première bouchée, je réalisai que la nourriture terrienne m'étais devenue insupportable, et je le jetai donc comme un fardeau inutile. Quelle ne fut pas alors ma stupeur lorsque je vis le pain en question, non seulement rester suspendu dans les airs, mais en plus - ô merveille ! - se mettre à décrire un cercle autour de moi! J'obtins ainsi une notion précise des véritables lois de la gravité, selon lesquelles tout corps mis en équilibre se meut en un cercle.

Autant j'avais été abattu à l'idée d'être devenu un jouet pour le destin railleur, autant je fus fier d'avoir été promu planète autonome, jouant qui plus est dorénavant le rôle de lune, et d'être compté au nombre des étoiles et des planètes de premier ordre. Et je dois reconnaître que dans ma vanité toute neuve, je fus véritablement pris d'un tel accès d'orgueil et d'euphorie, que si j'avais soudain vu tous les bourgmestres et leurs adjoints venir à ma rencontre, je les aurais toisés avec dédain comme des atomes, et n'aurais pas même daigné abaisser mon harpon sur eux.

Je demeurai dans cet état presque trois jours. Comme je gravitais autour de la fameuse planète, je pouvais aisément distinguer le jour de la nuit en voyant le soleil souterrain se lever et se coucher, même si leurs nuits ne sont pas comparables aux nôtres lorsque celuici disparaît. En effet, une fois que le soleil s'était couché, le ciel restait partout clair et lumineux, comme chez nous lorsque la lune est pleine ; et son lustre provenait apparemment de l'hémisphère de ce monde intérieur qui empruntait sa lumière au point milieu de ce soleil. Un minimum de connaissances en astronomie m'aidèrent à faire cette supposition.

Mais le meilleur reste à venir ! Alors que je rêvais béatement après les Dieux immortels, me considérant moi-même comme un nouvel astre appartenant à cette nouvelle cosmogonie, et comptant être rapidement repéré par les télescopes de la planète puis 
répertorié parmi les étoiles sans oublier mon satellite ; que vis-je ! Un horrible monstre ailé, qui m'attaqua, tantôt par la gauche, tantôt par la droite, tantôt de front et tantôt par derrière, me portant à chaque fois des coups se voulant mortels. En le voyant s'approcher, je crus d'abord qu'il s'agissait de l'un des douze signes du zodiaque, et j'espérais alors secrètement que c'était la Vierge, car aucun des autres ne pouvait alors mieux me consoler ni me distraire dans l'isolement qui était le mien. Mais lorsqu'il se trouva plus près, je vis qu'il ne s'agissait rien moins que d'un formidable griffon. Je fus tellement épouvanté que j'en oubliai qui j'étais et ce que j'étais devenu ; et dans cette panique indescriptible, je sortis mon Testimonium de l'Université que j'avais par chance dans ma poche, pour montrer à mon agresseur que j'avais à la fois mon certificat de théologie et de philosophie, que je poursuivais des études et qui plus est étais Bacalaureus ; bref, que j'étais en état de refouler toutes les revendications des opposants étrangers. Mais dès que mon premier accès de colère fut passé, je me ressaisis et ris de cette absurdité.

Je ne comprenais d'ailleurs pas pourquoi ce griffon me suivait. J'ignorais si je devais le considérer comme un ami ou un ennemi, ou, ce qui était plus vraisemblable, s'il ne voulait que m'observer pour assouvir sa curiosité. Il est vrai qu'un corps humain flottant dans les airs, un harpon à la main et une longue corde flottant derrière lui comme une longue queue, est un tableau susceptible d'attirer la curiosité de n'importe quel animal stupide. À propos de la forme peu ordinaire que je présentais alors, $\mathrm{j}$ 'appris plus tard qu'elle avait donné aux habitants de la planète voisine l'occasion de faire bon nombre de commentaires et suppositions. Les astronomes et les philosophes pensaient que j'étais une comète, et que la corde était sa queue. Certains prétendirent aussi qu'une trainée lumineuse inhabituelle comme celle-ci était le signe avant-coureur d'un malheur comme la peste, la famine ou quelque autre imprévu fâcheux. D'autres firent encore mieux et, aussi bien qu'ils le pouvaient de loin, reproduisirent très précisément ce que je représentais, si bien que j'étais déjà défini, décrit, peint et gravé sur du cuivre avant même d'être arrivé chez eux.

Plus tard, lorsque je serais descendu sur cette planète et que j'aurais appris la langue souterraine, je devrais trouver cette histoire très drôle et quelque peu flatteuse. Il faut dire aussi qu'au centre de la terre, il existe des étoiles inconnues de notre monde appelées Sciscisi - c'est-à-dire " chevelues " - que l'on dit affreuses à cause de leur chevelure rouge sang et hirsute comme des poils de brosse, et de leur traînée semblable à une longue barbe. Elles n'apparaissent jamais qu'occasionnellement, ce qui les classe donc comme chez nous parmi les présages célestes.

Mais revenons à notre histoire ! Le griffon s'était désormais approché au point de me frapper de ses ailes et d'essayer de dévorer l'une de mes jambes - je savais donc maintenant précisément dans quel but il me rendait visite. Je commençai dés lors à me défendre contre cet ennemi belliqueux ; je pris mon harpon à deux mains pour lui faire payer son audace, et au bout d'une heure et quelques essais infructueux, je ne parvins pas seulement à le faire fléchir, mais même à lui enfoncer mon harpon juste derrière la nuque, entre les deux ailes, au moment où il me saisissait, et avec une telle force que je ne réussis pas à le retirer. Le monstre poussa alors un cri affreux et fonça en direction de la planète qui se trouvait juste en-dessous de nous ; pour ma part, comme j'étais déjà fatigué de mon nouveau statut d'étoile, qui m'exposait apparemment à bien des dangers, comme c'est généralement le cas pour tout être supérieur : 
Je laissai donc l'oiseau m'entraîner vers la terre

On vit alors filer, brillant dans le ciel clair,

Une étoile semblant née du rayonnement solaire l'obscurité mais de l'absence de soleil, car il faisait encore si clair que je pouvais
distinguer toutes les lettres de mon Testimonium. Cette incandescence nocturne vient du
Firmament, c'est-à-dire de l'écorce terrestre interne de notre planète qui luit comme
notre lune. Pour ce qui est de la luminosité, il n'y a en conséquence aucune différence
entre le jour et la nuit, hormis le fait que le soleil est couché et que les nuits s'en trouvent
plus froides que les jours.

Les extraits de poésie latine proposés ci-dessous ont pour particularité d'être traduits non pas du latin, mais du danois : Jens Baggesen, en adaptant le texte latin d'Holberg, a en effet donné à lire à ses compatriotes non seulement le roman de son illustre devancier, mais également les citations de Virgile, Ovide, Horace ou Juvénal qui s'y trouvaient incluses.

Le texte français proposé ici constitue donc l'achèvement d'un long processus de migrations linguistiques : en s'attachant à conserver le rythme et la rime, il témoigne, par delà les changements de langues et d'époques, d'une fidélité sinon littérale, du moins littéraire à l'original classique.

La nuit tomba soudain sur le monde aux aguets ;

Un bruit sourd annonça ce que tous redoutaient;

La tempête éclata sous un ciel au supplice,

Imposant à la mer sa loi dévastatrice.

L'horizon retentit des grondements du tonnerre ;

Les cieux furent assaillis par des vagues en colère ;

Le bateau malmené espéra un répit,

Mais la mer s'acharna, et un cri d'agonie

Perça le ciel meurtri et la mer décousue ;

Un membre de l'équipage criait : « Nous sommes perdus! »

Virgile, L'Énéide III, 197.

N'aspire jamais à ce que le peuple inculte pense Ne comble pas celui que la bêtise dit immense ;

Le mica de la bêtise ne sait que jalouser !

Hélas! comme la sagesse peut vite s'évaporer

Dans le tourbillonnement des vagues du pouvoir

Qui poussent dans leur tourmente le bateau à s'échoir ;

Souvent, le poids des titres, de l'or et de l'argent

Font sombrer celui qui brilla de son vivant.

Rares sont les mortels qui ont reçu en cadeau

La tâche de soupeser de ce monde le terreau ;

Ils sont voués corps et âme, et à corps et à cri

À conserver leurs rangs, et tous leurs biens acquis,

Leur fierté empilée aura le dernier mot,

Pyramide inversée, érigée haut, très haut.

Les temps sont proches où elle viendra à s'effondrer

Retournant de ce monde au terreau sanctifié.

31 Horace, Épîtres I, 1 ; 34 ; 47. Juvénal, Satires X, 56 ; 103. 
Là coule le lait à flot, et l'hydromel aussi

Dont fraises et melons poussent de chaque côté du lit ;

Là vient jaillir du vin de Chypre des diamants

Pris dans la roche depuis des vagues de cristal ;

Là, côtoyant les roses de ce verdoyant val,

Honorant la rivière de son or scintillant.

Là et deci-delà, innombrables se tiennent,

Poussant en des milliers de parures rouges et vertes,

Les plus beaux fruits du Jardin de l'Éden,

Faisant signe d'approcher, les branches tendues, offertes ;

Là encore la potée mijote, et même près d'elle,

Une poule déjà plumée tomba soudain du ciel ;

Dans la marmite de jaspe vinrent se jeter tout droit

Une kyrielle bien dodue de lièvres sortis des bois ;

Ensuite sautèrent tous seuls à leur place sur le plat

Lagopèdes et bécasses et alouettes rôties,

Surgis de même avec des mines inassouvies,

Venus pour s'ajouter aux porcelets confits,

En parfaite symétrie, disposés sur le plat.

Une neige sucrée tomba, et du jus de citron

Se mit alors en glace; des tartes, des macarons

Devises et caractères : comment s'y prennent-ils donc?

Ni vu ni connu furent sur le plat, et d'un bond,

Pendant que les oiseaux qui n'étaient pas rôtis,

Tantôt un, tantôt deux, tantôt des nuées entières

Berçaient sans retenue du son de leur concert,

Le voyageur mangeant s'il en avait envie.

Ovide, Métamorphoses I, 111-12 ; 101-102.

L'homme qui n'a jamais faim oublie le goût des choses Jusqu'au parfum de l'art lorsqu'il imite les roses. Sa vie est comparable à un repas d'honneur Fait de plats raffinés qui lui soulèvent le cœur ; Pour qui la volupté est une notion sommaire, Les délices de la vie ont pris un goût amer, Et lorsque l'ennui ne cesse jamais un tant soit peu, L'esprit, dans son écorce, se meurt à petit feu.

Qui s'étonnerait de voir un cou large comme trois, Là-haut dans les sommets des Alpes enneigées? Qui pourrait être surpris de voir à Méroë, Des mamelles de la taille de l'enfant qui y boit? Qui pourrait sourciller à la vue des yeux bleus, Du front orné de cornes sous d'épais cheveux blonds Le long du Rhin, des fils des Germains belliqueux? Ce qu'ailleurs l'œil jamais ne pourra contempler, Extrêmement ordinaire s'avère en ces contrées, Où jamais personne ne saura s'en étonner.

Ainsi, gros comme le poing, un Pygmée aux jambes frêles Tient un glaive pas plus long que le doigt d'un humain, Et s'en va faire la guerre en marchant d'un bon train À l'armée formidable de guerriers pourvus d'ailes. Bientôt des grues cruelles font échouer son assaut, Saisissant dans ses griffes le minuscule héros, Puis le portent en triomphe dans les airs, haut, très haut. Chacun, chez nous, rirait à gorge déployée ; 
Nul habitant là-bas, à cette vue ne sourit

Et encore moins n'en rit, même si une telle armée,

Qu'elle soit la plus puissante, car y a enrôlé

De nombreux corps ailés, gradés et bien munis,

Malgré tout demeurera guère bien plus longue qu'un pied.

34 Juvénal, Satires XIII, 162.

Songez au grand péché que vous allez commettre,

Fuyez avant de perdre tout espoir de salut,

Surtout ne fermez pas votre âme à la vertu,

Ne laissez pas l'Amour devenir votre maître.

Pensez aux conséquences, si la toile qu'il vous tend,

Prend en toute perfidie votre esprit égaré ;

ô ! Comme disparaît vite l'envie de succomber,

Alors que le supplice demeure si lancinant.

Ovide, Métamorphoses VII, 70.

\section{NOTES}

1.Le qvamitique est la langue parlée par les Qvamites, qui sont les seuls véritables êtres humains du monde souterrain. (NdT)

2.L'Hekkenfeld est un glacier islandais. (NdT)

3.Ovide, Les Métamorphoses, livre II, 319.

4.Ovide, Les Métamorphoses, livre V, 423.

5.Virgile, L'Énéide, livre IV, 253.

6.Ovide, Les Métamorphoses, livre II, 202 et 320. 